\title{
Image Compression Radiography using HAAR Wavelet Transform
}

\author{
Swati Narula \\ M.tech, Manav Rachna International University \\ Faridabad
}

\author{
Sunanda Gupta \\ Manav rachna International University \\ Faridabad
}

\begin{abstract}
With significant increase in desire of multimedia machinery, the complication of strained bandwidth of a network and cache capacity arises. In fields such as Telemedicine, compact storage and adequate transmission of medical images is of bloom importance. Thus, a medical image abide to be compressed fore transmission. These have actuate the need for Image Compression. Image compression has become a necessity to ensure their storage and transfer in the network, while maintaining minimal time cost. In this paper we present a study and analysis of an image compression approach occupying on the principle of compression by Discrete Wavelet Transform (DWT). Haar wavelet transform based compression is one of the process that can be applied for compressing images. The method uses different specifications such as peak signal to noise ratio, mean squared error, \& signal to noise ratio to measure the performance and for comparing the results. This paper shows the objectives and methodology used for compressing different medical images and experimental results are presented and compared.
\end{abstract}

\section{Keywords:}

Image compression, Wavelet, Haar, Radiographs, Retained Energy.

\section{INTRODUCTION}

With the flourishing augmentation of technology and the entrance into the digital age, an ample expanse of image data must be handled to be saved in a proper way using efficient methods usually acquire in compressing images, while retaining high image quality and minimal reduction in image size [1]. The images play very considerable aspect in today's world. The image compression is used to save the storage space and network bandwidth which is the demand of today's environment. The uncompressed images crave the more storage space and bandwidth which slow down the activity of processor. The compressed image used lesser capacity of hard disk and easy to carry over internet without influencing the speed [2]. Compression is used because it helps to lessen the consumption of expensive resources such as hard disk space or transmission bandwidth. The basic goal of image data compression is to drain the bit rate for transmission and storage while either maintaining the original quality or providing an acceptable allegiance [3].Compression methods are being rapidly developed to compress large data files such as images, where data compression in multimedia applications has lately become more vital[4].

\subsection{Benefits of Compression}

- It provides a promising cost savings conjoining with sending less data over switched telephone network where cost of call is really usually based upon its continuation.

- It not only reduces vault requirements but also overall execution time.
- It also provides a level of shield against illicit monitoring [5].

\subsection{Classification of Compression}

There are two ways of classification of compression techniques- Lossless vs. Lossy compression:

In lossless compression schemes, the reconstructed image, posterior compression, is equivalent to the original image. In lossy schemes, the reconstructed image, later compression is not equivalent as the original image. It provide higher compression ratio [6].

\subsection{Image Compression Techniques}

A digital image, or "bitmap", abide of a grid of dots, or "pixels", with each pixel defined by a numeric value. Now, an appropriate piece of information may contain some portion which is not important and can be comfortably removed. All such data is referred as Redundant Data. Image compression aims at reducing the number of bits needed to represent an image by removing the spatial and spectral redundancies as much as possible. In general, three types of redundancy can be identified:

A. Coding Redundancy: If the gray levels of an image are coded in a way that uses enhanced code symbols than absolutely necessary to represent each gray level, the resulting image is said to contain coding redundancy.

B.Inter Pixel Redundancy: The Information of any given pixel can be reasonably anticipated from the value of its neighboring pixel. In order to reduce the inter pixel redundancies in an image, the 2-D pixel array normally used for viewing and apprehension must be transformed into a more adequate but usually 'non visual' format.

C.Psycho visual Redundancy: Certain information simply has less approximate importance than other information in general visual processing. This information is said to be Psychovisually redundant, it can be discarded without notably impairing the quality of image perception [7].

\subsection{Radiographs}

Radiographs are images conceived on a radiosensitive surface, such as photographic film, by radiation other than visible light, especially by $\mathrm{x}$-rays passed through an object [8]. Recently, tele radiology, which is one of the most used clinical aspects of telemedicine, attempts to deportation of medical images of various modalities, like computerized tomography (CT) scans, magnetic imaging (MRI), ultrasonography (US), and x-rays from one location to another such as in hospitals, imaging centers or a physician's desk. The radiological images are needed to be compressed afore transmission to a distant location or due to the bandwidth or storage restrictions [9]. 


\section{WAVELETS}

A wave is a fluctuating function of time or space and is periodic. In contrast, wavelets are localized waves [10]. Wavelet means a "small waves". The smallness implies to a window function of finite length. Wavelets are mathematical tools for stratified decomposing functions. Wavelet Transform has been proved to be a very propitious tool for image processing in modernistic years to get the compressed images at higher compression ratios with higher PSNR values. It is a prominent transform used for some of the image compression standards in lossy compression methods. Unlike the discrete cosine transform, the wavelet transform is not Fourier-based and therefore wavelets do a better job of handling discontinuities in data. Wavelets are mathematical functions which help in representing the original image into an image in frequency domain, which can else be divided into sub band images of different frequency components [11].

Wavelet transform sections the data of an image into approximation (low frequencies) and detail (high frequencies) sub-signals. The approximation (LL) sub-signal shows the general trend of pixel values and other three detail sub-signals show the vertical (LH), horizontal (HL) and diagonal (HH) details or changes in the images. Splitting of signal into two parts shown in Figure 1. A simple example of level 3 decomposing is shown in Figure 2[12].



Figure 1:- Splitting of signals into two parts



\begin{tabular}{|l|l|l|}
\hline LL2 & HL2 & \multirow{2}{*}{ HL1 } \\
\cline { 1 - 2 } LH2 & HH2 & \\
\hline \multicolumn{2}{|c|}{ LH1 } & HH1 \\
\hline
\end{tabular}

\begin{tabular}{|l|c|c|c|}
\hline LL3 & HL3 & \multirow{2}{*}{ HL2 } & \multirow{2}{*}{ HL1 } \\
\cline { 1 - 2 } LH3 & HH3 & \\
\cline { 1 - 2 } LH2 & HH2 & \\
\hline \multicolumn{3}{|c|}{ LH1 } & HH1 \\
\hline
\end{tabular}

Third level

\begin{tabular}{l|l}
$\begin{array}{l}\text { Most } \\
\text { important } \\
\text { part of } \\
\text { the image }\end{array}$ & $\begin{array}{c}\text { Second } \\
\text { level }\end{array}$ \\
\hline & $\begin{array}{l}\text { First } \\
\text { level }\end{array}$
\end{tabular}

Figure 2:- Levels of DWT Decomposition

\subsection{Picture Quality Measures of Image Compression}

Normally the peculiarity of an image compression scheme can be deliberated in premise of Distortion measurement criterions [13].

Distortion measurement: At present, the utmost extensively used objective distortion measures are the MSE and the related PSNR.

The MSE is defined by:-

MEAN SQUARE ERROR $($ MSE $)=$

$1 / N \sum M \sum N\left(x j, k-x^{\prime} j, k\right) 2$ for $\mathrm{J}=1$ and $\mathrm{K}=1$

Where $\mathrm{M}$ denotes number of columns and $\mathrm{N}$ number of rows $x j, k-x^{\prime} j, k$ denotes pixel values of original image before compression and degraded image after compression.

The smaller value of MSE, the better compressed image represents the original image. Mathematically the PSNR is given in decibels as:

PEAK SIGNAL TO NOISE RATIO (PSNR) =

$10 \log \left(2^{\wedge} \mathrm{n}-1\right)^{\wedge} 2=10 \log 255^{\wedge} 2$

$$
\text { MSE MSE }
$$

\subsection{Haar Wavelet Transform}

Analyses of wavelets begins with HAAR wavelet, the pioneer and elementary. Haar wavelet enumerate a wavelet

transform to represent image. It is the basic transformation from space to a local frequency domain. A HT disintegrate each signal into two components, one is called average (approximation) or trend and the other is known as difference (detail) or fluctuation. This process is repeated repeatedly [14].

The Properties of the Haar Transform are described as follows:

i) Haar Transform is real and orthogonal.

$\mathrm{Hr}=\mathrm{Hr}^{*}(1)$

$\mathrm{Hr}=\mathrm{Hr}(2)$ 
ii) The basis vectors of the Haar matrix are consecutively organized.

iii) Haar Transform has poor energy compaction for images.

iv) Orthogonally: The original signal is split into a low semifinal matrix $(\mathrm{T})$ whose rows and columns have a high frequency part and filters enabling the diverging without replicating information are said to orthogonal.

v) Linear Phase: To obtain linear phase, symmetric filters would have to be used.

vi)Compact support: The magnitude response of the filter should be exactly zero outside the frequency range covered by the transform. If this property is satisfied, the transform is energy invariant.

vii) Perfect reconstruction: If the input signal is transformed and inversely modified using a set of weighted basis functions and the reproduced sample values are equivalent to those of the input signal, the transform is said to have the perfect reconstruction property. If, in addition no information redundancy is present in the sampled signal, the wavelet transform.is, as declared above a, ortho normal [15]

\section{PROPOSED METHODOLOGY}

The announce algorithm is particularized as follows:

1. Input medical image (e.g.:- CT SCAN, MRI, USG and XRAY) of size of $256 \times 256 \times 8$ at gray scale (X).

Table illustrating the results of image compression using haar for MRI CERVICAL SPINE is shown in table 1 . The wavelet based image compression for level $(\mathrm{N})=1$ and threshold value $=5$ is shown in figure 4.2. Enter decomposition level $(\mathrm{N})$ that gives the wavelet decomposition of the matrix input image at level $\mathrm{N}$.

3. Enter Haar wavelet family (W)

4. Find the DWT coefficients (approximation and detail) of original image. Approximate details are same as original image details. Horizontal details construct only horizontal information (edges).Vertical details construct only vertical information (edges). Diagonal details construct very few information of input image. So approximation image is applied into next Level for deformation.

5. Enter the local threshold value (T).

6. Decompose the approximate coefficient to $\mathrm{N}$ level by W.

7. Reconstruct the matrix $\mathrm{X}$ based on the multi-level wavelet decomposition structure.

8. Find the Retained Energy (PERF2) in the compressed image.

9. Compute Mean Square Error (MSE).

10. Compute Peak Signal to Noise Ratio (PSNR). [16]

\section{SIMULATION RESULTS AND DISCUSSIONS}

In order to assess the performance of the advised admittance of image compression using Haar wavelet transform technique three standard medical images are being considered. The work is implemented using MATLAB. The evaluation of the proposed approach in image compression was performed and the following results are inferred:-

The database of the medical images that are acknowledged for compression has been shown in Figure 3[17].

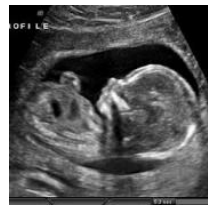

(a)

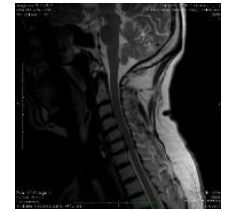

(b)

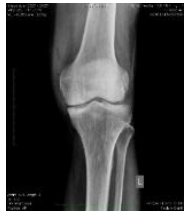

(c)
Figure 3:- Database of medical radiographs

(a) USG OVARY

(b) MRI CERVICAL SPINE

(c) XRAY KNEE

Table 1:- Showing results of image compression for MRI CERVICAL SPINE

\begin{tabular}{|c|c|c|c|c|c|c|c|c|c|}
\hline $\begin{array}{l}\text { LEVEL } \\
\text { DECOMPOSITION } \\
\text { (N) }\end{array}$ & $\begin{array}{l}\text { GLOBAL } \\
\text { POSITIVE } \\
\text { THRESHOL } \\
\text { D }\end{array}$ & $\begin{array}{l}\text { PERCENTAG } \\
\text { E OF } \\
\text { WAVELET } \\
\text { COEFICIENT } \\
\text { S SET TO } \\
\text { ZER0:PERFO }\end{array}$ & $\begin{array}{l}\text { PERCENTAGE OF } \\
\text { IMAGE ENERGY } \\
\text { PRESERVED:PERF } \\
\text { L2 }\end{array}$ & $\begin{array}{l}\text { PSNR VALUE } \\
\text { FOR } \\
\text { DECOMPRESSIO } \\
\text { N IMAGE USING } \\
\text { WAVELET } \\
\text { COMPRESSION }\end{array}$ & $\begin{array}{lr}\text { THE } & \text { TIME } \\
\text { NEED } & \text { TO } \\
\text { EXECUTE } & \\
\text { THIS } & \text { WBC } \\
\text { ONLY } & \end{array}$ & $\begin{array}{l}\text { ORIGINAL } \\
\text { IMAGE SIZE }\end{array}$ & $\begin{array}{l}\text { COMPRESSED } \\
\text { IMAGE SIZE }\end{array}$ & MSE_WO & $\begin{array}{l}\text { SIGNAL } \\
\text { TO NOISE } \\
\text { RATIO } \\
\text { (SNR) }\end{array}$ \\
\hline \multirow[t]{4}{*}{1} & 5 & 71.1559 & 99.9635 & 49.7152 & 26.4844 & 986102 & 60484 & 0.6943 & 28.4173 \\
\hline & 8 & 72.9856 & 99.9357 & 49.0679 & 27.0625 & 986102 & 58752 & 0.8059 & 27.7700 \\
\hline & 10 & 73.5438 & 99.9188 & 48.9160 & 27.9688 & 986102 & 58174 & 0.8346 & 27.6182 \\
\hline & 15 & 74.2692 & 99.8783 & 48.6999 & 29.4375 & 986102 & 57456 & 0.8772 & 27.4021 \\
\hline \multirow[t]{4}{*}{2} & 5 & 85.5172 & 99.9461 & 47.7356 & 28.5469 & 986102 & 58434 & 1.0953 & 26.4378 \\
\hline & 8 & 88.5623 & 99.8995 & 46.6749 & 27.3750 & 986102 & 55330 & 1.3983 & 25.3770 \\
\hline & 10 & 89.5963 & 99.8681 & 46.2921 & 27.7344 & 986102 & 54750 & 1.5271 & 24.9942 \\
\hline & 15 & 91.0342 & 99.7865 & 45.9550 & 27.7844 & 986102 & 54030 & 1.6504 & 24.6571 \\
\hline 3 & 5 & 88.0576 & 99.9415 & 47.1575 & 28.0469 & 986102 & 57410 & 1.2512 & 25.8596 \\
\hline
\end{tabular}




\begin{tabular}{|l|l|l|l|l|l|l|l|l|l|}
\hline & 8 & 91.5082 & 99.8886 & 45.7486 & 24.6406 & 986102 & 54462 & 1.7307 & 24.4507 \\
\hline & 10 & 92.7225 & 99.8518 & 45.1331 & 27.8125 & 986102 & 53722 & 1.9942 & 23.8353 \\
\hline & 15 & 94.4830 & 99.7516 & 44.2996 & 28.8125 & 986102 & 52836 & 2.4161 & 23.0018 \\
& & & & & & & & & \\
\hline 4 & 5 & 88.4700 & 99.9406 & 47.0592 & 28.8438 & 986102 & 57898 & 1.2799 & 25.7613 \\
\hline & 8 & 92.0037 & 99.8865 & 45.5931 & 29.1719 & 986102 & 55078 & 1.7938 & 24.2953 \\
\hline & 10 & 93.2584 & 99.8484 & 44.9079 & 24.3281 & 986102 & 54096 & 2.1003 & 23.6101 \\
\hline & 15 & 95.0988 & 99.7435 & 43.8523 & 23.7813 & 986102 & 53812 & 2.6782 & 22.5545 \\
& & & & & & & & & \\
\hline 5 & 5 & 88.5421 & 99.9412 & 47.0479 & 29.6406 & 986102 & 61204 & 1.2832 & 25.7501 \\
\hline & 8 & 92.0884 & 99.8875 & 45.5546 & 27.7031 & 986102 & 55016 & 1.8097 & 24.2568 \\
\hline & 10 & 93.3479 & 99.8497 & 44.8615 & 26.5313 & 986102 & 55756 & 2.1229 & 23.5637 \\
\hline & 15 & 95.1991 & 99.7454 & 43.6829 & 35.2031 & 986102 & 53930 & 2.7848 & 22.3850 \\
\hline
\end{tabular}
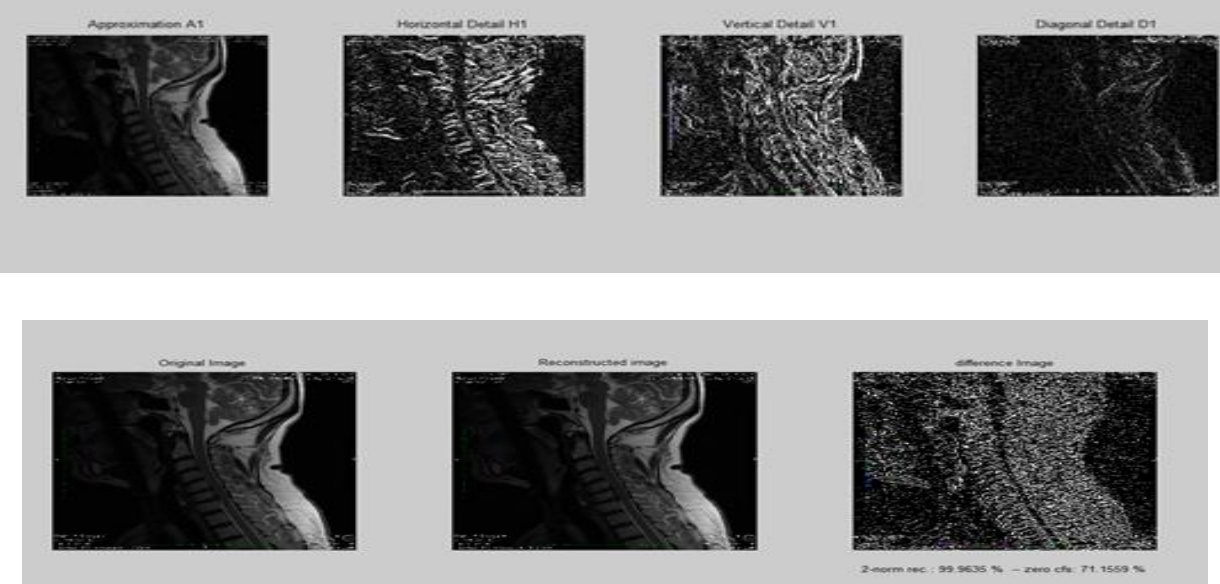

Figure 4:- Wavelet based image compression for MRI CERVICAL SPINE

Table 2:- showing results of image compression for USG OVARY.






\begin{tabular}{|l|l|l|l|l|l|l|l|l|l|}
\hline & 8 & 60.2308 & 99.9148 & 39.0423 & 5.5313 & 88562 & 4182 & 8.1068 & 14.2386 \\
\hline & 10 & 65.8236 & 99.8643 & 37.0102 & 4.5781 & 88562 & 3982 & 12.9439 & 12.2065 \\
\hline & 15 & 75.6746 & 99.6955 & 33.5196 & 4.3438 & 88562 & 3756 & 28.9151 & 8.7159 \\
\hline 5 & 5 & 48.2352 & 99.9732 & 43.6701 & 4.6719 & 88562 & 4622 & 2.7930 & 18.8664 \\
\hline & 8 & 60.2470 & 99.9211 & 39.0421 & 3.7969 & 88562 & 4182 & 8.1072 & 14.2384 \\
\hline & 10 & 65.8364 & 99.8743 & 37.0100 & 4.4688 & 88562 & 3982 & 12.9443 & 12.2063 \\
\hline & 15 & 75.6834 & 99.7181 & 33.5196 & 4.7344 & 88562 & 3756 & 28.9151 & 8.7159 \\
\hline
\end{tabular}

Table illustrating the results of image compression using haar for USG OVARY is shown in table 2. The wavelet based image compression for level $(\mathrm{N})=1$ and threshold value $=5$ is shown in figure 5 .
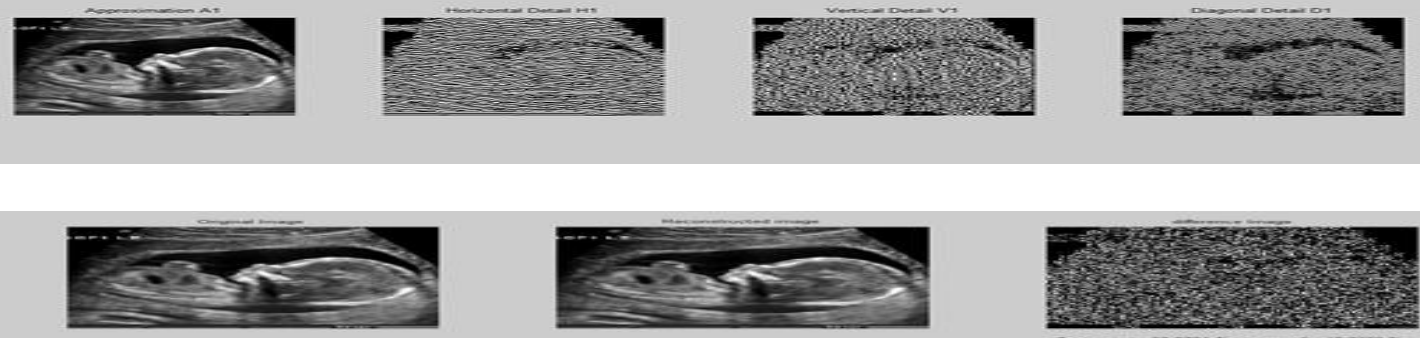

Figure 5:- Wavelet based image compression for USG OVARY.

Table 3:- showing results of image compression for XRAY KNEE.

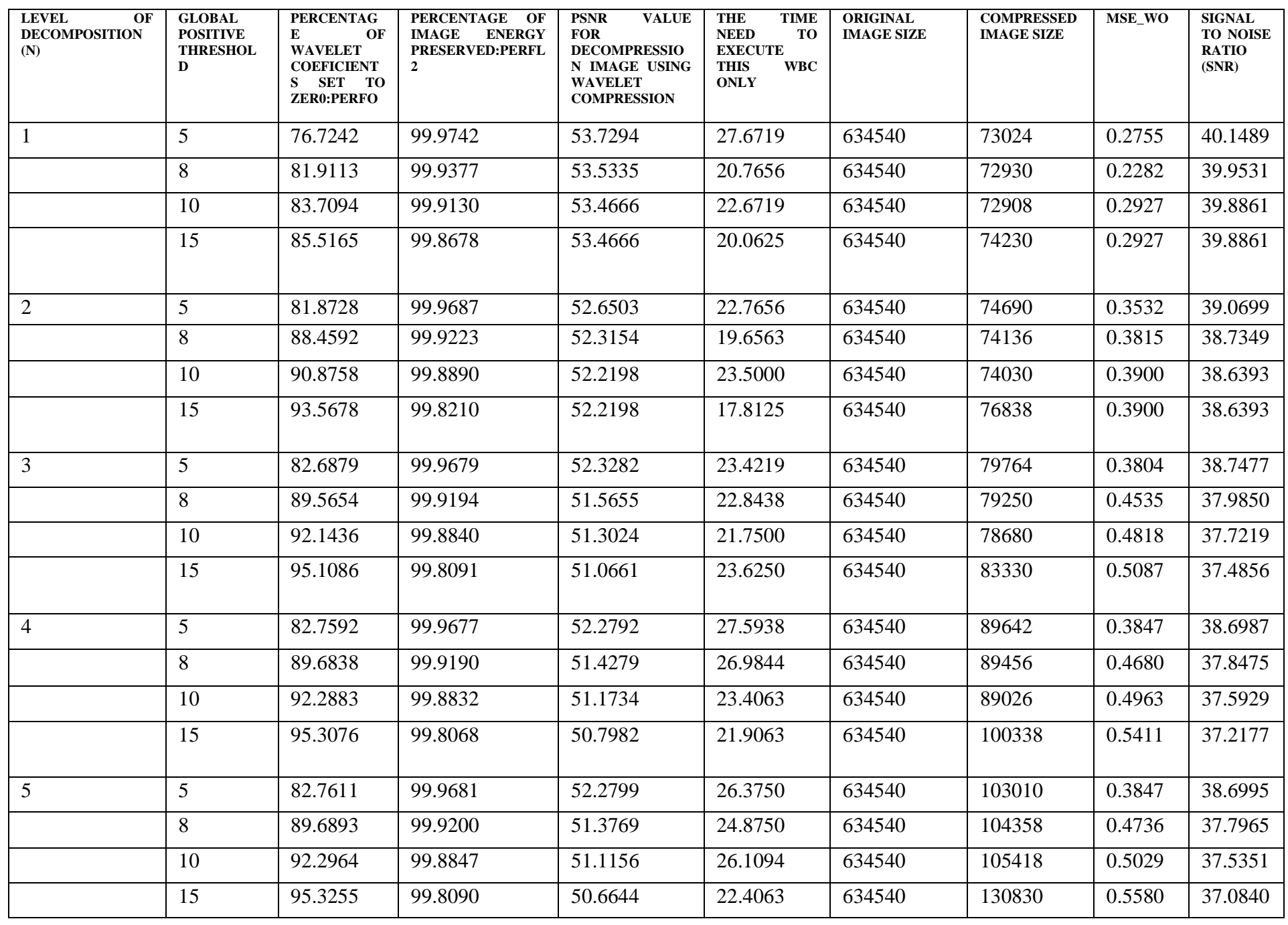


Table showing the illustrating of image compression using haar for XRAY KNEE is shown in table 3. The wavelet based image compression for level $(\mathrm{N})=1$ and threshold value $=5$ is shown in figure 6 .

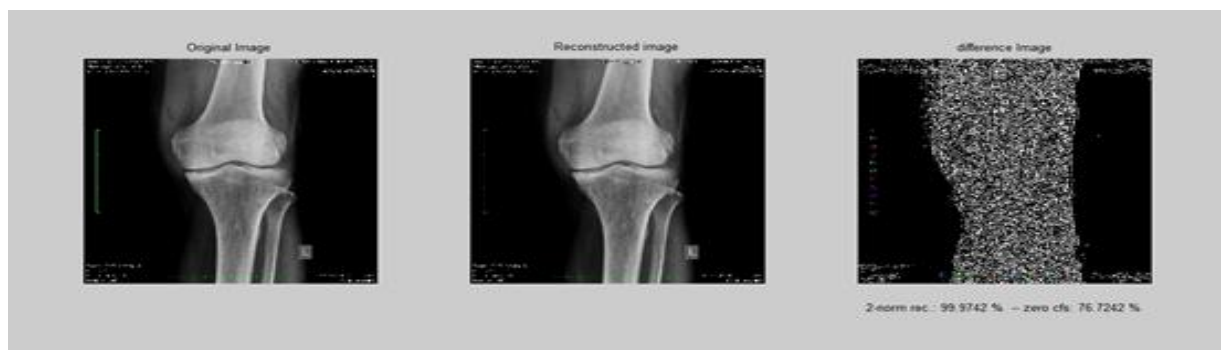

Figure 6:- Wavelet based image compression for XRAY KNEE.

\section{CONCLUSION}

A peculiar approach to medical radiograph Compression employing a haar wavelet is schemed in this paper. In this work we adduce the Comparative study of wavelet based compression quite successfully using different images. XRAY KNEE has maximum Peak Signal To Noise Ratio (PSNR) as compared to other Medical Images and USG OVARY image has been compressed to a larger extent i.e. high compression ratio $(\mathrm{CR} \%)$ and has maximum Mean Square Error as compared to other Medical Images. In future this can be extended by considering other discrete wavelet transforms such as Biorthogonal and symlets wavelet and analyzing which results in better compression ratio and high PSNR.

\section{ACKNOWLEDGEMENT}

I would like to acknowledge and extend my appreciativeness to the following persons who have made the completion of this research paper possible. I am very thankful to, Ms. Sunanda Gupta, for her encouragement, support and for providing the facilities for the completion of this paper. This work would not have been possible without the encouragement and her guidance. Most especially to my family and friends. And to God, who made all things possible.

\section{REFRENCES}

[1] K. Ratakonda and N. Ahuja, "Lossless Image Compression with Multiscale Segmentation", IEEE Transactions Image Processing, vol. 11, no.11, 2002, pp. 1228-1237.

[2] Rajwinder Kaur and Sheenam Malhotra, "Compression of Image with Haar Wavelet and Neural Network A Review".

[3] Neeraj Singla and Sugandha Sharma , "A Review on Wavelet based Compression using Medical Images", International Journal of Innovative Research in Computer and Communication Engineering Vol. 1, Issue 8, October 2013.

[4] M. J. Nadenau, J. Reichel, and M. Kunt, "Wavelet Based Color Image Compression: Exploiting the Contrast Sensitivity Function", IEEE Transactions Image Processing, vol. 12,no.1, 2003, pp. 58-70.

[5] Neeraj Singla and Sugandha Sharma , "A Review on Wavelet based Compression using Medical Images", International Journal of Innovative Research in Computer and Communication Engineering Vol. 1, Issue 8, October 2013.
[6] Rajwinder Kaur and Sheenam Malhotra, "Compression of Image with Haar Wavelet and Neural Network A Review".

[7] K. H. Talukder and K. Harada, "Haar Wavelet Based Approach for Image Compression and Quality Assessment of Compressed Image", IAENG International Journal of Applied Mathematics, 2007.

[8] Singh S., Kumar V., and Verma H.K., Adaptive Threshold-based block classification in medical image.

[9] Adnan Khashman, and KamilDimililer, "Image Compression using Neural Networks and Haar Wavelet," WSEAS Transactions on Image Processing, Vol. 4, no. 5, Pp. 330-339, 2008.

[10] Abood Kuther, Aboud Hayder, and A.H. Falih, "X-ray image compression using neural network," ISSN 22295518, Vol.3, Issue 10, 2012.

[11] K. H. Talukder and K. Harada, "Haar Wavelet Based Approach for Image Compression and Quality Assessment of Compressed Image", IAENG International Journal of Applied Mathematics, 2007.

[12] Kamrul Hasan Talukder and Koichi Harada, "Wavelet Based Approach for Image Compression and Quality Assessment of

[13] Compressed Image.” Proceeding in IAENG International Journal of Applied Mathematics in Feb 2007.

[14] Divya Prashar and Archana Kumar, "A Review on Enhancement in Compression of Radiograph Image Using Wavelets and Neural Network".

[15] Ajay K.S, Shamik Tiwari and V.P. Shukla, "Wavelet based Multi Class image classification using Neural Network," International Journal of Computer Applications, vol.37, no.4, 2012.

[16] Sonja Grgic, Mislav Grgic, Member, 2001. IEEE and Branka Zovko-Cihlar, Member, IEEE , "Performance Analysis of Image Compression Using Wavelets",

[17] IEEE Trans., Vol: 48.

[18] B.Nassiri, R.Latif1, A .Toumanari1, A. Bssis, S.Elouaham, K.EL mansouri, F. Maoulainine, "Study of Wavelet Based Medical Image Compression Techniques”, ISSN: 2319-5967 ISO 9001:2008 Certified International Journal of Engineering Science and Innovative Technology (IJESIT) Volume 3, Issue 3, May 201418

[19] Radiographs were taken from a radiological department of the medical institute from a medical practitioner. 